\title{
Epigenetic role of nuclear S6K1 in early adipogenesis
}

\author{
Sang Ah Yi, Jihoon Han \& Jeung-Whan Han ${ }^{*}$ \\ Research Center for Epigenome Regulation, School of Pharmacy, Sungkyunkwan University, Suwon 16419, Korea
}

\begin{abstract}
S6K1 is a key regulator of cell growth, cell size, and metabolism. Although the role of cytosolic S6K1 in cellular processes is well established, the function of S6K1 in the nucleus remains poorly understood. Our recent study has revealed that S6K1 is translocated into the nucleus upon adipogenic stimulus where it directly binds to and phosphorylates H2B at serine 36. Such phosphorylation promotes EZH2 recruitment and subsequent histone H3K27 trimethylation on the promoter of its target genes including Wnt6, Wnt10a, and Wnt10b, leading to repression of their expression. S6K1-mediated suppression of Wnt genes facilitates adipogenic differentiation through the expression of adipogenic transcription factors PPAR $y$ and Cebpa. White adipose tissues from S6K1-deficient mice consistently exhibit marked reduction in H2BS36 phosphorylation (H2BS36p) and H3K27 trimethylation (H3K27me3), leading to enhanced expression of Wnt genes. In addition, expression levels of H2BS36p and H3K27me3 are highly elevated in white adipose tissues from mice fed on high-fat diet or from obese humans. These findings describe a novel role of $\mathrm{S6K1}$ as a transcriptional regulator controlling an epigenetic network initiated by phosphorylation of H2B and trimethylation of $\mathrm{H} 3$, thus shutting off Wnt gene expression in early adipogenesis. [BMB Reports 2016; 49(8): 401-402]
\end{abstract}

Ribosomal protein S6 kinase 1 (S6K1), a key downstream effector of the mammalian target of rapamycin (mTOR), plays a critical role in protein synthesis, lipid synthesis, cell growth,

*Corresponding author. E-mail: jhhan551@skku.edu

http://dx.doi.org/10.5483/BMBRep.2016.49.8.116

Received 30 June 2016

Keywords: Adipogenesis, Epigenetics, EZH2, Histone modification, S6K1

Abbreviations: EZH2, enhancer of zeste homolog 2; H2BS36p, histone H2B serine 36 phosphorylation; H3K27me3, histone H3 lysine 27 trimethylation; mTOR, mammalian target of rapamycin; S6K1, ribosomal protein $\mathrm{S} 6$ kinase 1

Perspective to: Sang Ah Yi et al (2016), S6K1 Phosphorylation of H2B Mediates EZH2 Trimethylation of H3: A Determinant of Early Adipogenesis, Molecular Cell, May 5;62(3):443-52, doi: 10.1016/ j.molcel.2016.03.011. and aging. To understand the molecular mechanism of S6K1-mediated cellular processes, most S6K1 research studies have focused on the identification of downstream targets of S6K1. Recent reports have revealed that S6K1 phosphorylates carbamoyl-phosphate synthetase 2, aspartate transcarbamylase, and dihydroorotase (CAD), explicating S6K1-dependent cell proliferation and DNA synthesis. Another unique substrate of S6K1 is BMAL1, a circadian transcription factor associated with translational machinery in the cytosol. It promotes protein synthesis by S6K1-mediated phosphorylation. While most substrates of S6K1 have been found in the cytoplasm, nuclear substrates of S6K1 have not yet been clearly identified. Although there is some evidence showing nuclear translocation of p70 S6K1, nuclear function of S6K1 has been rarely supported experimentally. Some limited reports have revealed that S6K1 phosphorylates transcription factors including cAMP-response-element modulator $\tau$ (CREM $\tau)$ and oestrogen receptor $\alpha$ at serine 167 . Our recent paper has demonstrated that nuclear S6K1 acts as a direct histone modifier that can phosphorylate histone $\mathrm{H} 2 \mathrm{~B}$ at serine 36 (S.A. $\mathrm{Yi}$ et al. Mol Cell, 2016, 62(3):443-52). The level of phosphorylated $\mathrm{H} 2 \mathrm{~B}$ at serine 36 is increased upon adipogenic stimulus accompanied by the activation and nuclear translocation of S6K1. Even though the mechanism underlying the translocation of S6K1 into the nucleus remains unknown, our recent study has identified a nuclear substrate of S6K1 for the first time.

It has been reported that many signaling kinases can phosphorylate histone molecules. For example, MSK1/2, PKB/Akt, and RSK2/p90 S6K2 belonging to AGC kinase family can phosphorylate histone $\mathrm{H} 3$. Phosphorylation at each distinct residue of histone by these kinases affects other histone modifications, thereby generating an epigenetic network. Interestingly, p70 S6K1, another member of the AGC kinase family, share similar target motif with the histone kinases. Based on our recent study, S6K1, like other AGC kinase family members, can also phosphorylate $\mathrm{H} 2 \mathrm{~B}$ at serine 36 during adipogenesis. Such phosphorylation is required for EZH2 recruitment that can facilitate further methylation associated with gene suppression. In this manner, a combination of histone modifications constitutes a code for binding of other proteins, thus altering chromatin structure and regulating gene transcription.

Histone crosstalk falls into two categories. First, it has been frequently observed that histone phosphorylation has an 


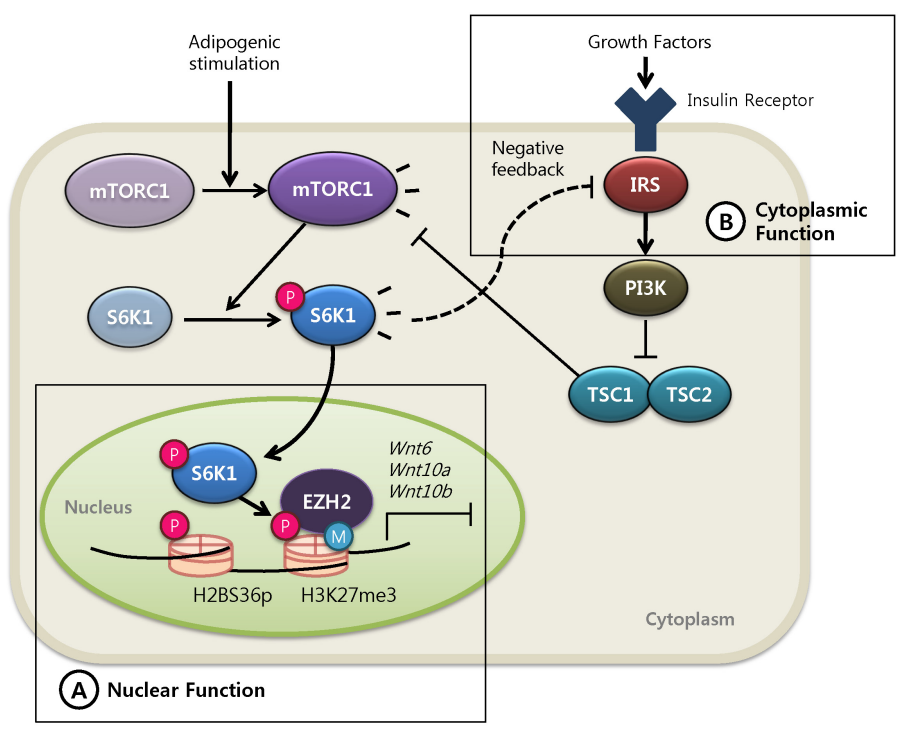

Fig. 1. Schematic model of metabolic regulation by $\mathrm{S} 6 \mathrm{~K} 1$ in two ways. (A) Upon adipogenic stimulation, activated S6K1 moves into the nucleus and phosphorylates H2BS36, further inducing EZH2 recruitment and $\mathrm{H} 3 \mathrm{~K} 27$ trimethylation. This epigenetic network suppresses the expression of Wnt6, 10a and $10 \mathrm{~b}$ genes, leading to adipogenic commitment. (B) While growth factormediated activation of insulin receptor substrate (IRS) activates mTOR-S6K1 pathway through a series of downstream signaling molecules, activated S6K1 phosphorylates and inhibits IRS, operating a negative feedback loop in the cytoplasm. impact on adjacent modifications within the same histone tail ('cis' effects). For instance, phosphorylation of histone $\mathrm{H} 3$ at serine or threonine residue promotes the acetylation of nearby lysine residue of histone $\mathrm{H} 3$, thus regulating gene transcription. The second type of histone crosstalk occurs between two different histone molecules ('trans' effects). For example, modification of histone $\mathrm{H} 2 \mathrm{~B}$ by mono-ubiquitination induces the methylation of histone $\mathrm{H} 3$, specifically H3K4 and H3K79. While histone phosphorylation is known to mainly induce intra-histone crosstalk, we have shown that phosphorylation of histone $\mathrm{H} 2 \mathrm{~B}$ at serine 36 by S6K1 induces methylation of histone $\mathrm{H} 3$ at lysine 27 , thereby suppressing the transcription of $\mathrm{EZH} 2$ target genes. These results suggest that histone phosphorylation is possibly involved in inter-histone crosstalk as well as intra-histone crosstalk.

It has been demonstrated that S6K1-deficient mice are lean at birth and resistant to high fat diet (HFD)-induced obesity. This anti-obesity phenotype of S6K1-deficient mice stems from impaired early adipogenesis. However, the molecular mechanism underlying S6K1-mediated promotion of early adipogenesis is unknown. Our findings have demonstrated that stimulus for adipogenic commitment promotes S6K1 activation and nuclear translocation. Activated S6K1-mediated H2BS36p and H3K27me3 suppress the expression of Wnt6, Wnt10a, and Wnt10b genes, thus facilitating early adipogenesis. Considering the fact that obesity results from the increase in the number and size of adipocyte, our study suggests that S6K1-mediated epigenetic network might be used as a therapeutic target for treating obesity.

Several reports have implicated that S6K1 is involved in not only adipogenesis, but also glucose homeostasis, insulin sensitivity, and lipolysis (M. Pende et al. Nature, 2000, 408(6815):994-997; S.H. Um et al. Nature, 2004, 431(7005): 200-205). These findings suggest that S6K1 could regulate metabolic homeostasis in a complex way. Our recent findings, together with previous reports, have allowed us to refine the molecular model of S6K1-dependent metabolic regulation (Fig. 1). S6K1-mediated transcriptional regulation is apparently independent of its known functions in the cytoplasm. Dual roles of S6K1 in two distinct subcellular locations offer a potential therapeutic avenue for treating obesity and insulin resistance, the hallmark of type 2 diabetes. Future identification of new nuclear targets of S6K1 might be able to explain other phenotypes of S6K1-deficient mice, including hypothalamic control of energy homeostasis, muscle atrophy, renal hypertrophy, memory impairment, and extended lifespan.

\section{ACKNOWLEDGEMENTS}

This work was supported by a grant (NRF-2012R1A5A2A 28671860) of the Medical Research Center Program through the National Research Foundation of Korea (NRF) funded by the Ministry of Education, Science and Technology, Republic of Korea. 